\title{
4 AÇÕES TERAPÊUTICAS PARA PESSOAS COM ESQUIZOFRENIA ACOMPANHADAS NUM CENTRO DE ATENÇÃO PSICOSSOCIAL
}

| Marciana Moll1'; Josiane Alves²; Daniela Silva ${ }^{3}$; Thiago Faria4; Carla Ventura ${ }^{5}$; Lucas Duarte Silva ${ }^{6}$ |

\section{RESUMO}

CONTEXTO: Atualmente, a esquizofrenia tem se mostrado um transtorno que provoca um crescente número de prejuízos psicossociais ao sujeito, e mudanças à rotina da família.

OBJETIVO: Investigar as ações terapêuticas para pessoas com diagnóstico de esquizofrenia em tratamento em um Centro de Atenção Psicossocial.

METODOLOGIA: Investigação interpretativa, cuja coleta de dados se deu por meio da entrevista semi-estruturada com a equipe técnica do referido serviço. Os dados foram analisados por meio de análise temática.

RESULTADOS E CONCLUSÕES: Entre as ações terapêuticas realizadas, se destacam os atendimentos psicoterápicos e as oficinas terapêuticas grupais. Entre essas oficinas a geradora de renda foi considerada como um meio de aceitação do portador de transtorno mental na comunidade. Assim, é importante que o enfermeiro estimule a participação das pessoas com diagnóstico de esquizofrenia nessas oficinas e que sejam elaborados novos estudos referentes às atividades terapêuticas que promovem a reabilitação de portadores de esquizofrenia.

\section{PALAVRAS-CHAVE: Enfermagem; Esquizofrenia; Transtornos mentais; Serviços comunitários de saúde mental}

\section{RESUMEN}

"Acciones terapéuticas para personas con esquizofrenia acompañadas en um Centro de Atención Psicosocial"

CONTEXTO: En la actualidad, la esquizofrenia ha sido un trastorno que causa un número creciente de daño psicosocial al individuo, y cambia la rutina de la familia.

OBJETIVO: Investigar las acciones terapéuticas en personas con esquizofrenia en tratamiento en un Centro de Atención Psicosocial.

MATERIAL Y MÉTODOS: Investigación interpretativa, que la recolección de datos fue a través de entrevistas semi-estructuradas con el equipo técnico el servicio. Los datos fueron analizados mediante análisis temático.

RESULTADOS Y CONCLUSIONES: Entre las acciones terapéuticas realizadas, destacamos las citas psicológicas y grupales talleres terapéuticos. Entre ellas se encuentra el ingreso talleres de generación se consideró como un medio de la aceptación de los enfermos mentales en la comunidad. Por lo tanto, es importante que las enfermeras animan a la participación de las personas con esquizofrenia en estos talleres y se elaboran nuevos estudios relacionados con las actividades terapéuticas que promueven la rehabilitación de los pacientes esquizofrénicos.

DESCRIPTORES: Enfermería; Esquizofrenia; Trastornos mentales; Servicios comunitarios de salud mental

\begin{abstract}
“Therapeutic actions for people with schizophrenia under treatment in a Psychosocial Care Center"

BACKGROUND: Currently, schizophrenia has been a disorder that causes an increasing number of psychosocial damage to the subject, and changes the family's routine.

AIMS: Investigate the therapeutic actions for people with schizophrenia undergoing treatment in a Psychosocial Care Center.

METHODS: Interpretive research was held; which data was collected through semi-structured interviews with the technical team of the service. Data were analyzed using thematic analysis.

RESULTS AND CONCLUSIONS: Among the therapeutic actions performed, we highlight the psychological appointments and group therapeutic workshops. Among these, the income generating workshops were considered as a means of acceptance of mental patients in the community. Thus, it is important for nurses to encourage the participation of people with schizophrenia in these workshops and the elaboration of further studies related to therapeutic activities that promote rehabilitation of schizophrenic patients.
\end{abstract}

KEYWORDS: Nursing; Schizophrenia; Mental disorders; Community mental health services

Submetido em 20-02-2015

Aceite em 30-05-2015

1 Enfermeira; Doutora em Ciências; Professora na Universidade de Uberaba, Campus Aeroporto, 38055-500 Uberaba/Minas Gerais, Brasil, mrcna13@yahoo.com.br 2 Enfermeira; Especialista em Enfermagem em Unidade de Tratamento Intensivo; Enfermeira Assistencial na Clínica Médica do Hospital de Clínicas da Universidade Federal do Triângulo Mineiro, 38040-500 Uberaba/Minas Gerais, Brasil, josifatureto@hotmail.com

3 Enfermeira; Especialista em Urgência e Emergência; Enfermeira Responsável Técnica da Clínica de Vacinação Imunize, Uberaba/Minas Gerais, dannyenfer@hotmail.com 4 Enfermeiro; Especialista em Enfermagem em Unidade de Tratamento Intensivo; Enfermeiro Assistencial no Bloco Cirúrgico do Mário Palmério Hospital Universitário da Universidade de Uberaba, 38050-501 Uberaba/Minas Gerais, Brasil, josifatureto@hotmail.com

5 Advogada; Mestre em Direito e Doutora em Administração; Professora Associada na Universidade de São Paulo, Escola de Enfermagem de Ribeirão Preto, 14040-902 Ribeirão Preto - São Paulo, Brasil, caaventura@gmail.com

6 Graduando em Enfermagem na Universidade de Uberaba, 38030-390 Uberaba/Minas Gerais, Brasil, lucasduarteslv@hotmail.com

Citação: Moll, F. M., Alves, F. J., Silva, D. C., Faria, T. D. S., Ventura, C. A. A., \& Silva, L. D. (2015). Ações terapêuticas para pessoas com esquizofrenia acompanhadas em um Centro de Atenção Psicossocial. Revista Portuguesa de Enfermagem de Saúde Mental (14), 24-30. 


\section{INTRODUÇÃO}

O termo esquizofrenia inicialmente foi denominado demência precoce, pois iniciava na juventude e tinha um prognóstico variável, podendo evoluir para o predomínio de sintomas incapacitantes, tais como: embotamento afetivo, isolamento social e dificuldades cognitivas, alteração de atenção, compreensão e catatonia (Generoso, 2008).

Bleuler e Kraepelin foram figuras importantes na evolução histórica da esquizofrenia. Contudo, constata-se certa dicotomia em seus estudos, uma vez que Bleuler privilegiava os sintomas e não o curso e o resultado. Nessa perspectiva, esse estudioso enfatizava a alteração da capacidade de associação do pensamento e não o processo de deterioração, o que originou o termo esquizofrenia. Já Kraepelin defendia a ideia de que todas as pessoas acometidas pela demência precoce sofreriam de um processo de deteriorização, além de não valorizar os sintomas (Câmara, 2007).

Posteriormente, surgiram outros estudiosos de relevância para este tema como o psiquiatra francês Benedict A. Morel que fez o uso do termo démense précoce em indivíduos com a associação do transtorno psicótico e quadros de deterioração com início na adolescência. Karl Ludwing Kahlbaum enfatizou os sintomas da catatonia e Ewold Hecker discorreu sobre o comportamento bizarro da hebefrenia (Sadock, Sadock, 2007).

A partir desta trajetória histórica dos estudos relacionados à esquizofrenia e a sua evolução, surgiram diversas definições para o termo. Dentre essas definições, destacou-se a que esclarece que a esquizofrenia é uma alteração do pensamento considerada uma doença do cérebro grave e duradoura, gerando comportamentos psicóticos e diversas dificuldades das quais se destacam a de processar informações e de relacionamento interpessoal e com o mundo externo (Lobo, Mattiolli, Santos, 2008).

Do mesmo modo, Araripe Neto, Bressan e Busatto Filho (2007) expõem que a esquizofrenia é uma desorganização entre o pensamento e a realidade que predispõe a anormalidades no convívio social.

$\mathrm{Na}$ atualidade, a esquizofrenia vem se tornando um problema de saúde que ocasiona danos psicossociais na vida do indivíduo como: dificuldades no auto cuidado e no processamento de informações, bem como nas relações interpessoais, o que favorece o isolamento social e consequentemente dificulta o processo de reabilitação desses sujeitos (Ferreira Junior, Barbosa, Barbosa, Hara, Rocha, 2010).
Desse modo, é importante, ainda, citar que a esquizofrenia tende a desencadear um sofrimento familiar, pois este transtorno psicótico ocasiona mudanças na rotina da família por exigir cuidados contínuos. Ainda, a existência de estigmas sociais acerca dos transtornos mentais também dificulta a aceitação da família (Almeida, Schal, Martins, Modena, 2010).

Sendo assim, o tema é relevante para a saúde, e estudos sobre ele são necessários para instrumentalizar as equipes multiprofissionais com informações técnicas e científicas que possibilitem a elaboração de estratégias que minimizem a evolução da referida patologia e ofereçam apoio aos familiares.

A partir dessas considerações, esse estudo objetivou descrever as ações terapêuticas de um Centro de Atenção Psicossocial direcionadas para indivíduos com diagnóstico de esquizofrenia.

De acordo com a Portaria 336/2002 do Ministério da Saúde, o CAPS deve ser composto por uma equipe multiprofissional composta por médico psiquiatra e profissionais de nível superior pertencentes as seguintes categorias profissionais: psicólogo, assistente social, enfermeiro, terapeuta ocupacional, pedagogo ou outro profissional necessário ao projeto terapêutico. Nessa equipe devem existir, também, profissionais de nível médio como auxiliar ou técnico de enfermagem, técnico administrativo e artesão. Segundo Nascimento e Galvanese (2009), toda essa equipe deve oferecer uma assistência aos usuários de forma humanizada, proporcionando um cuidado integral em saúde mental que proporcione a reabilitação e reinserção psicossocial dos usuários desse serviço.

Nesse contexto, ressalta-se a importância dos profissionais de saúde elaborarem estudos com este enfoque, uma vez que os profissionais que atuam nos CAPS têm maior proximidade junto ao portador desse transtorno psicótico e seus familiares durante as atividades recreativas, orientações, a administração de medicações, dentre outras ações compatíveis às propostas terapêuticas da atualidade. Essa realidade proporciona um estreitamento do vínculo entre a equipe multidisciplinar e o portador de esquizofrenia e seus familiares, o que favorece a essa equipe elaborar ações terapêuticas que possibilitem a adesão desses sujeitos ao tratamento. 


\section{METODOLOGIA}

Trata-se de pesquisa do tipo descritivo de caráter exploratório e com abordagem qualitativa. A descrição e exploração possibilitam a identificação de problemas, estabelecendo relações entre as variáveis que, à luz da análise teórica, aprimoram o conhecimento do objeto de estudo.

Os dados foram coletados após a aprovação do projeto pelo Comitê de Ética de Pesquisa em Seres Humanos da Universidade de Uberaba. A coleta de dados se deu por meio da entrevista semiestruturada, com profissionais do CAPS estudado, realizada nos meses de agosto e setembro de 2011, no próprio Centro de Atenção Psicossocial, conforme agendamento determinado por cada um dos participantes. Antes de obtermos os dados, foi proporcionada aos participantes a oportunidade de apreciar os objetivos do estudo, os riscos e os benefícios.

Os questionamentos que nortearam a coleta dos dados pertinentes ao objetivo desse estudo se embasaram nos seguintes aspectos: as ações terapêuticas realizadas no Centro de Atenção Psicossocial e a contribuição dessas ações para a reabilitação e reinserção social dos portadores de esquizofrenia. Para participar do estudo, esses sujeitos deveriam se enquadrar nos seguintes critérios de inclusão: profissionais integrantes da equipe técnica do referido CAPS; estar trabalhando no serviço há, no mínimo, dois anos e ter concluído a graduação em sua área de formação, há, no mínimo, um ano. Aos profissionais que estavam inseridos nesses critérios foi solicitado o consentimento informado. A escolha desses critérios se deu por considerarmos que o tempo mínimo de atuação profissional no CAPS por dois anos favorece a obtenção de informações fidedignas, pois estes membros da equipe técnica já têm experiência na área de saúde mental e também na instituição referida.

O estudo foi realizado em um Centro de Atenção Psicossocial que presta cuidados humanizados e está cadastrado no Ministério da Saúde há mais de dez anos, o que nos faz identificar que a referida instituição está em concordância com os preceitos da Portaria 336/2002. Sendo assim, este CAPS pode ser considerado um cenário de estudo rico para a obtenção de informações que podem contribuir para o meio científico. Vale destacar que neste serviço existem treze membros da equipe técnica, os quais se enquadraram nos critérios de inclusão já citados. No entanto, foram entrevistados dez desses profissionais, pois três deles não se interessaram em participar da pesquisa.
Para a análise dos dados, as fitas gravadas durante as entrevistas foram transcritas na íntegra pelos próprios pesquisadores. Inicialmente, foi realizada uma primeira leitura para se obter visão geral do conteúdo obtido. Posteriormente, foi realizada análise temática dos dados para que se pudesse efetivar a discussão dos dados. Para tanto, utilizou-se como embasamento os seguintes passos: ordenação dos dados, classificação dos dados e categorias de análise (Minayo, 2007).

A análise dos dados permitiu a identificação das seguintes categorias de análise: ações multidisciplinares para estabilizar os sintomas da esquizofrenia e oficinas terapêuticas de caráter produtivo.

\section{RESULTADOS}

\section{Ações Multidisciplinares para Estabilizar os Sintomas da Esquizofrenia}

Os participantes do estudo informaram que no Centro de Atenção Psicossocial são realizadas as seguintes ações pela equipe multidisciplinar, com vistas à estabilização dos sintomas da esquizofrenia: atendimento psicoterápico, terapia em grupo e tratamento medicamentoso. [...] fazemos grupos com pessoas que tem problemas semelhantes, sem rotular ninguém [...]. (G)

As atividades são: oficinas terapêuticas (higiene e hábitos de vida, música, dança, boneca, artesanato), assembléia, grupos de psicoterapia e consulta médica. $(\mathrm{H})$ Dentre as atividades citadas pelos entrevistados, os participantes enfatizaram o atendimento psicoterápico, que consiste em atividades individuais ou em grupos, realizadas de acordo com a necessidade dos indivíduos assistidos, através das técnicas de psicoterapia.

[...] o paciente pode ficar na terapia ocupacional, a psicoterapia todos fazem, é um pacote e todos são inseridos nele [...]. (F)

[...] cada usuário aqui tem um terapeuta de referência, eles têm um grupo e eles fazem parte da psicoterapia. E tem um terapeuta de referência na casa por semana [...]. (D)

As propostas de atendimento nos Centros de Atenção Psicossocial priorizam ações realizadas por uma equipe multidisciplinar, o que faz com que as terapias em grupos se destaquem dentre as atividades propostas nesses serviços. Essa realidade foi expressa pelos entrevistados, conforme relatos abaixo.

[...] mas também tem as oficinas fechadas, eles participam muito dessas oficinas fechadas com a psicóloga. E as oficinas de terapia ocupacional, que é para um público 
específico, não que sejam da mesma patologia, sinais e sintomas não, mas um grupo que tenha interesse de personalidade, de gosto, alguma coisa que se identificam. E definidas essas oficinas, forma-se um grupo no semestre, naquele semestre vamos supor que formou um grupo de sete pessoas, vão essas sete pessoas do começo ao fim [...]. (B)

Nos grupos participam sempre um psicólogo e um enfermeiro ou um psicólogo e um assistente social, de acordo com as necessidades mais importantes. Mas tem sempre dois profissionais diferentes [...]. (C)

Diante as ações realizadas no Centro de Atenção Psicossocial, a terapia medicamentosa contribui efetivamente para a eliminação e/ou estabilização de sintomas, sendo esta atividade expressa pelos entrevistados como uma ação terapêutica de suma importância.

É, a gente não pode negar primeiro a ação medicamentosa que através da ação medicamentosa, não especificamente, mas quase sempre que a gente consegue reverter o quadro [...]. (F)

Tendo em vista as diferentes ações terapêuticas mencionadas pelos participantes, identificamos que esse Centro de Atenção Psicossocial busca diversificar as propostas terapêuticas com vistas à reabilitação do portador de transtorno mental, independente do diagnóstico médico dos usuários do serviço.

\section{Oficinas Terapêuticas de Caráter Produtivo}

No Centro de Atenção Psicossocial são desenvolvidas atividades que buscam inserir os usuários na comunidade e na produção de produtos que podem ser comercializados. Dentre essas oficinas destacaram-se as oficinas terapêuticas de caráter produtivo e a arteterapia. Nesse contexto, existe um destaque para as práticas de oficinas grupais com um enfoque na produção de bens para o consumo e desenvolvimento de novas habilidades.

Dentre as atividades relatadas que seguem a proposta da economia solidária pode-se citar: oficina de sabão, sacola, boneca, mosaico, crochê, bordar blusas, chinelos e panos de prato. Também as oficinas de trabalho corporal, de hábitos de vida e higiene pessoal foram mencionadas pelos participantes:

[...] as atividades terapêuticas são realizadas nas oficinas, tendo as oficinas de arteterapias que são coordenadas pela arteterapeuta [...]. (G)

[...] a gente tenta fazer com que eles participem das oficinas terapêuticas, tem também oficinas de música que é eu que faço, tem oficina de dança, oficina de boneca [...]. (H)
[...] tem oficinas de economia solidária que a gente montou tem essa de boneca que nós fazemos e estamos começando a partir de semana que vem oficinas de flor de tecido [...]. (C)

Dessa forma, percebe-se que as oficinas terapêuticas trazem a possibilidade de a comunidade atentar-se ao trabalho daqueles indivíduos com transtorno mental, valorizando-os e compreendendo a importância do convívio com as diferenças.

\section{DISCUSSÃO}

De acordo com os entrevistados, o atendimento psicoterápico se destacou e consiste em atividades individuais ou em grupos que são realizados por meio de técnicas de psicoterapia, considerando-se a necessidade dos indivíduos assistidos. Para Santos, Duarte (2009), esta estratégia terapêutica possibilita que o profissional identifique os problemas individuais de cada usuário devido ao estreitamento das relações entre o psicólogo e o usuário, facilitando a elaboração de um plano terapêutico pertinente para atender as necessidades biopsicossociais de cada sujeito.

Por meio do atendimento psicoterápico, o psicólogo identifica as necessidades biopsicossociais dos portadores de transtornos mentais, o que permite a elaboração de intervenções embasadas nas necessidades desse indivíduo e dos seus familiares, bem como a sua inserção nos planos terapêuticos existentes nos Centros de Atenção Psicossocial (intensivo, semi-intensivo e não intensivo). Outro aspecto benéfico que resulta do atendimento psicoterápico nos Centros de Atenção Psicossocial é o estreitamento do vínculo entre o usuário e o profissional de saúde. Nos dias atuais, a terapia em grupo é caracterizada como um recurso terapêutico benéfico na assistência em saúde, principalmente na saúde mental, pois favorece a ressocialização do indivíduo em sofrimento mental (Benevides, Pinto, Cavalcante, Jorge, 2010). Benevides et al. (2010) ainda esclarecem que deve-se valorizar alguns aspectos relacionados à formação de um grupo, tais como: a seleção dos participantes, a avaliação das condições de cada membro selecionado e a programação das atividades que serão realizadas. Ressalta-se, portanto, que a vivência em grupo possibilita o senso de inclusão, valorização e identificação nas experiências coletivas dos problemas de saúde. Além disso, desenvolve novos valores como a escuta. Ainda, diante de várias pessoas com a mesma problemática, a capacidade de enfrentamento dos participantes aumenta (Schrank, Olschowsky, 2008). 
Assim, o trabalho em grupo (re)compõe a autonomia de portadores de transtorno mental e os possibilita a transitar por outros serviços em busca da promoção de saúde. Nesse contexto, estudo que aborda a utilização de serviços de saúde da família revela que portadores de transtorno mental desenvolvem dependência de serviços especializados (Nascimento, Brêdo, Santos, Lima, 2013). Nessa perspectiva, os participantes da pesquisa enfatizaram a terapia em grupo como possibilidade de fomentar o senso de inclusão nos indivíduos que nela se inserem, uma vez que os participantes do grupo tendem a ser considerados diferentes no meio social. A partir dessa realidade, este tipo de terapia proporciona momentos de livre expressão de pensamentos e sentimentos, o que a faz ser um instrumento propulsor da ressocialização de indivíduos com transtornos mentais. Com vistas à eliminação e/ou estabilização de sintomas, o tratamento medicamentoso foi expresso pelos entrevistados como uma atividade importante. Essa ação terapêutica se dá por meio da prescrição de medicamentos, administração e orientações para os usuários e seus familiares. De acordo com Rinaldi, Bursztyn (2008), a prescrição medicamentosa é realizada pelo médico psiquiatra e as demais ações relacionadas ao tratamento medicamentoso (administração e orientações) são desempenhadas pelos enfermeiros e demais membros da equipe de enfermagem que, também, são responsáveis por elaborar estratégias para o uso correto dessa medicação prescrita, o que previne novas crises.

Além disso, o sucesso da terapia medicamentosa permite o acompanhamento clínico e a reinserção social destes indivíduos na sociedade, como exemplo, acesso ao trabalho, atividades de lazer, exercício dos direitos civis e fortalecimento dos laços familiares e comunitários (Santos, Duarte, 2009). Também foi relatado por todos os participantes que a ação mais favorável para a terapêutica dos indivíduos com diagnóstico de esquizofrenia é a oficina. Nesse sentido, Schrank, Olschowsky (2008) afirmam que as atividades realizadas nas oficinas permitem a autonomia dos participantes no meio social e enfatizam a importância da cidadania. As oficinas terapêuticas incluem o tratamento e a reabilitação do usuário, demonstrando-se assim, como uma experiência positiva no âmbito de possibilidades de exercício de cidadania. Constituem, ainda, possibilidade de estímulo à preparação para o mercado de trabalho, permitindo que o usuário retorne às suas atividades e também desvende habilidades novas, conseguindo consolidar sua autonomia (Mielke, Kantorski, Jardim, Olschowsky, Machado, 2009).
Dessa forma, as oficinas buscam constituir nos indivíduos com diagnóstico de esquizofrenia a promoção de laços de cuidado, trabalho e afetos com as outras pessoas (Schrank, Olschowsky, 2008).

Cabe também lembrar que, na perspectiva do novo modelo de atenção psicossocial proposto desde o início do movimento de reforma psiquiátrica, passou-se a valorizar os grupos terapêuticos. Gigante (2011) explicita que, dentre esses grupos, existem as oficinas terapêuticas que visam abordar diversas dimensões da vida humana, das quais se destacam: psicológico, motor, criatividade, capacidade intelectual, entre outros aspectos, com ênfase nas necessidades individualizadas dos sujeitos, embora se priorize as atividades coletivas. No âmbito da saúde mental, Lussi, Pereira (2011) observam que as oficinas terapêuticas contribuem como uma nova alternativa de tratamento, pois as atividades terapêuticas com fins laborais podem produzir materiais geradores de renda.

A geração de renda acontece de maneira indireta, pois muitos participantes utilizam as oficinas para aprenderem a produzir bens (sabão, bonecas, entre outros) e após aprenderem, muitos deles os produzem, por conta própria e, posteriormente os comercializam informalmente em feiras livres. Observa-se que a inserção ou reinserção de pessoas que apresentam transtornos mentais em atividades de trabalho, mesmo que informalmente, tem ocorrido pela oficina geradora de renda. Porém, apesar de algumas iniciativas de estruturação de cooperativas no âmbito da saúde mental, estas ainda não se tornaram uma realidade (Lussi, Pereira, 2011). A dificuldade em consolidar cooperativas que absorvam as produções advindas das referidas oficinas foi evidenciada nesta investigação, pois os usuários geralmente a utilizam apenas para aprendizado. Há, também, um destaque para a arteterapia que agrega conhecimentos de diversas áreas, o que a caracteriza como uma atividade transdisciplinar que objetiva conhecer o indivíduo de forma integral, por meio de atividades de autoconhecimento e transformação. Desse modo, pode-se definir a arteterapia como uma atividade que trabalha com a criatividade e a utiliza como uma maneira de comunicação (Coqueiro, Vieira, Freitas, 2010). Nessa perspectiva, muitos usuários que têm dificuldades de se comunicar verbalmente, conseguem expor seus pensamentos por meio das artes, o que permite aos profissionais de saúde mental avaliá-los quanto a aspectos culturais, afetivos, cognitivos, motores e sociais, dentre outros. Para Coqueiro et al. (2010), a arte é vista como um instrumento que permite revelar a potencialidade do sujeito. 
São vários os tipos de arteterapias, sendo que dentro de uma instituição fica a critério do arteterapeuta definir o cronograma das atividades a serem realizadas, de acordo com as necessidades da clientela assistida (Coqueiro et al., 2010). Gigante (2011) afirma que as experiências trocadas durante as oficinas geradoras de renda (tal como a arteterapia e oficina de sabão, entre outras) agregam valores de cooperação, capacidade de autonomia, igualdade, solidariedade, autoestima e responsabilidade para contribuir no resgate da cidadania destes indivíduos.

\section{CONCLUSÃO}

De maneira geral, as ações terapêuticas de um Centro de Atenção Psicossocial voltadas para indivíduos com diagnóstico de esquizofrenia foram representadas pelas oficinas terapêuticas, atendimento psicoterápico, terapia em grupo e individual, tratamento medicamentoso, arteterapia, terapia familiar e consultas psiquiátricas. Deve-se enfatizar que as ações apontadas como àquelas que são essenciais para os indivíduos com diagnóstico de esquizofrenia foram a psicoterapia e as oficinas com caráter de geração de renda, mas também foram citadas a arteterapia e o tratamento medicamentoso.

Com base nessa realidade, parece que as ações terapêuticas de serviços comunitários e especializados em saúde mental (CAPS) devem centrar-se em ações grupais, as quais devem ser subsidiadas pelos princípios da Reforma Psiquiátrica brasileira que prioriza a desinstitucionalização do portador de transtorno mental e a promoção de ações que não visem apenas o diagnóstico médico e sim a necessidade de interação social do ser humano, bem como a consolidação de seu direito ao exercício da cidadania. Por fim, é relevante reforçar a importância de que sejam valorizadas as necessidades de cada indivíduo, o que pode ter uma relação direta com a sintomatologia e o prognóstico do transtorno mental com vistas à elaboração de ações que favoreçam a reabilitação e, consequentemente a inclusão social dessa clientela.

\section{REFERÊNCIAS BIBLIOGRÁFICAS}

Almeida, M. M., Schal, V. T., Martins, A. M., e Modena, C. M. (2010). A sobrecarga de cuidadores de paciente com esquizofrenia. Revista de Psiquiatria do Rio Grande do Sul, 32(3), 73-79. doi: 10.1590/S010181082010005000003
Araripe Neto, A. G. A., Bressan, R. A., e Busatto Filho, G. (2007). Fisiopatologia da esquizofrenia: Aspectos gerais. Revista de Psiquiatria Clínica, 34(Supl 2), 198203. Acedido em 17 de novembro de 2014 em http:// www.hcnet.usp.br/ipq/revista/vol34/s2/198.html

Benevides, D. S, Pinto, A. G. A, Cavalcante, C. M., e Jorge, M. S. B. (2010). Cuidado em saúde mental por meio de grupos terapêuticos de um hospital-dia: Perspectivas dos trabalhadores de saúde. Interface comunicação, saúde, educação, 14(32), 127-138. doi: 10.1590/ S1414-3283201000010001

Câmara, F. P. (2007). A catástrofe de Kraepelin. Revista Latinoamericana de Psicopatologia Fundamental, 10(2), 307-318. Acedido em 20 de novembro de 2014 em http://www.fundamentalpsychopathology.org/art/ jun2007/fernando.pdf

Coqueiro, N. F., Vieira, F. R. R., e Freitas, M. M. C. (2010). Arteterapia como dispositivo terapêutico em saúde mental. Acta Paulista de Enfermagem, 23(6), 859862. doi: $10.1590 /$ S0103-21002010000600022

Ferreira Junior, B. C., Barbosa, M. A., Barbosa, I. G., Hara, C., e Rocha, F. L. (2010) Alterações cognitivas na esquizofrenia: Atualização. Revista de Psiquiatria do Rio Grande do Sul, 32(2), 57-63. doi: 10.1590/S010181082010000200006

Generoso, C. M. (2008). O funcionamento da linguagem na esquizofrenia: Um estudo lacaniano. Ágora, 11(2), 267-281. doi: 10.1590/S1516-14982008000200007

Gigante, M, P. (2011). Perfil das oficinas de geração de trabalho e renda no âmbito da atenção psicossocial no Brasil. Dissertação. Universidade Católica de Pelotas, Pelotas, Brasil.

Lobo, G. O., Mattiolli, T. C., e Santos, S. A. (2008). Esquizofrenia: Perspectiva histórica e assistência de enfermagem. Cuidarte Enfermagem, 2(2), 192-203. Acedido em 17 de novembro de 2014 em http://www.fundacaopadrealbino.org.br/facfipa/ner/pdf/ed03enfpsite.pdf

Lussi, I. A. O., e Pereira, M. A. O. (2011). Empresa social e economia solidária: Perspectivas no campo da inserção laboral de portadores de transtorno mental. Revista da Escola de Enfermagem da USP, 45(2), 515-521. doi: 10.1590/S0080-62342011000200030 
Mielke, F. B., Kantorski, L. P., Jardim, V. M. R., Olschowsky, A., e Machado, M. S. (2009). O cuidado em saúde mental no CAPS no entendimento dos profissionais. Ciência e Saúde Coletiva, 14(1), 159-164. doi: 10.1590/S1413-81232009000100021

Minayo, M. C. S. (2007). O desafio do conhecimento: Pesquisa qualitativa em saúde. São Paulo: Hucitec.

Nascimento, A. F., e Galvanese, A. T. C. (2009). Avaliação da estrutura dos centros de atenção psicossocial do município de São Paulo, SP. Revista de Saúde Pública, 43(Supl 1), 8-15. doi: 10.1590/S003489102009000800003

Nascimento, Y. C. M. L., Brêda, M. Z., Santos, R. M., e Lima, K. R. S. (2013). Concepções sobre a identidade social da pessoa em sofrimento mental nos estudos de enfermagem. Cogitare Enfermagem, 18(1), 102108. Acedido em 20 de fevereiro do 2015 em http:// ojs.c3sl.ufpr.br/ojs/index.php/cogitare/article/viewFile/31314/20021
Portaria no 336/2002 de 19 de fevereiro. Diário Oficial da União. Ministério da Saúde.

Rinaldi, D. L., e Bursztyn, D. C. (2008). O desafio da clínica na atenção psicossocial. Arquivos Brasileiros de Psicologia, 60(2), 32-39. Acedido em 10 de fevereiro de 2015 em http://seer.psicologia.ufrj.br/index.php/abp/ article/view/243/203

Sadock, B. J., \& Sadock, V. A. (2007). Compêndio de psiquiatria: Ciências do comportamento e psiquiatria clínica. Porto Alegre: Artes Médicas.

Santos, T. M., e Duarte, M. (2009). A atuação do psicólogo no contexto do centro de atenção psicossocial de álcool e drogas da Prefeitura de Uberlândia. União Educacional de Minas Gerais. Acedido em 20 de fevereiro de 2015 em http://www.psicologia.pt/artigos/ textos/TL0159.pdf

Schrank, G., e Olschowsky, A. (2008). O centro de atenção psicossocial e as estratégias para inserção da família. Revista da Escola de Enfermagem da USP, 42(1), 127-134. doi: 10.1590/S0080-62342008000100017

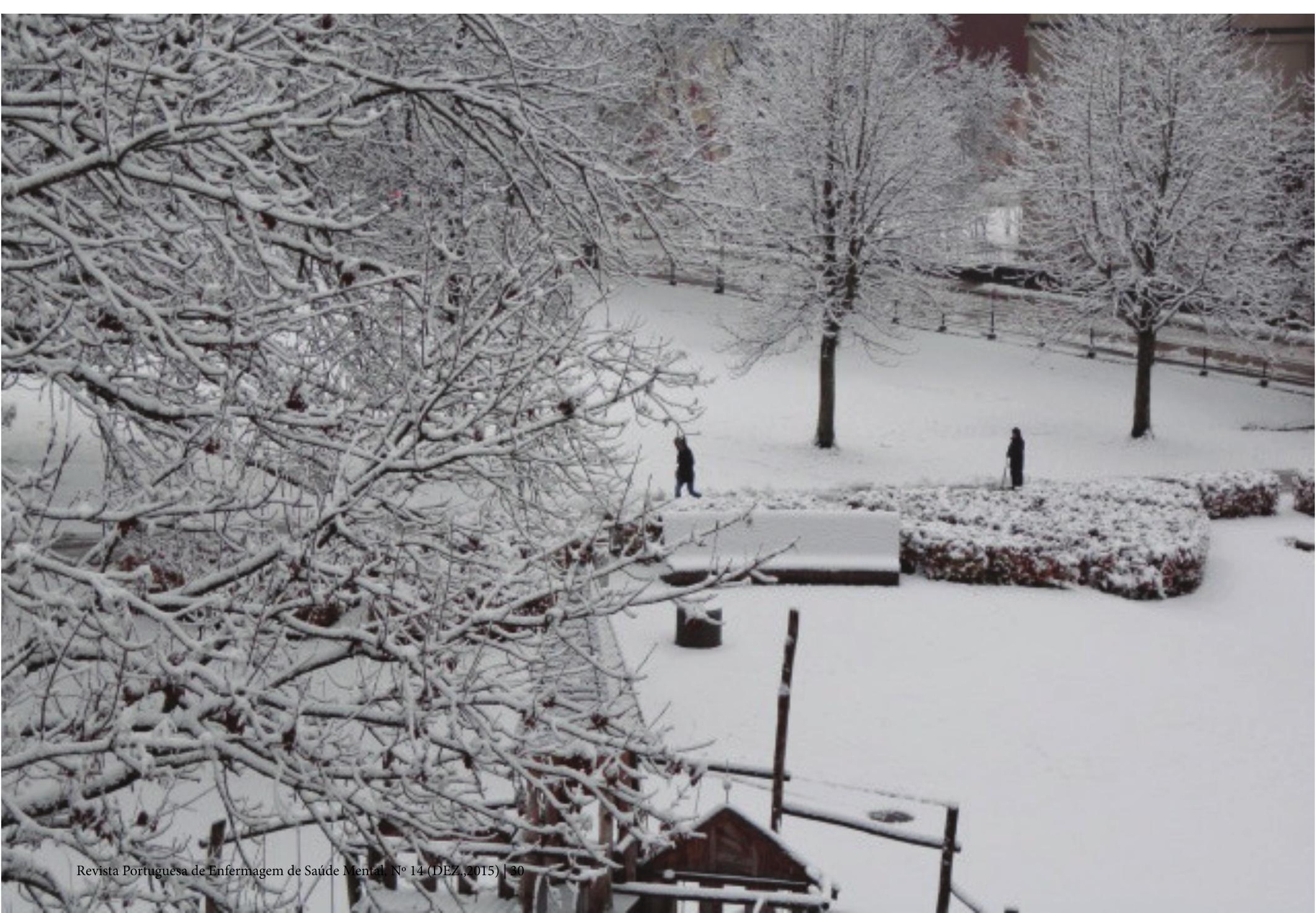

